\title{
Évaluation Du Niveau D'efficacité Technique Des Systèmes De Production A Base De Maïs Au Bénin
}

\author{
Toléba Séidou Mamam \\ Ingénieur Agronome, DEA, Chercheur en Agronomie, \\ Département d'Économie, de Socio-Anthropologie et de Communication \\ pour le développement rural, Faculté des Sciences Agronomiques / \\ Université d'Abomey-Calavi, Cotonou, Bénin \\ Biaou Gauthier \\ Professeur Titulaire, Agroéconomiste, Département d'Économie, \\ de Socio-Anthropologie et de Communication pour le développement rural, \\ Faculté des Sciences Agronomiques / Université d'Abomey-Calavi.

\section{Zannou Afio} \\ Maître Assistant, Département d'Économie, de Socio-Anthropologie et \\ de Communication pour le développement rural, Faculté des Sciences \\ Agronomiques / Université d'Abomey-Calavi, Cotonou, Bénin.
}

\section{Saïdou Aliou}

Maître de Conférences, Département de Production Végétale, Faculté des Sciences Agronomiques / Université d'Abomey-Calavi, Cotonou, Bénin.

doi: 10.19044/esj.2016.v12n27p276 URL:http://dx.doi.org/10.19044/esj.2016.v12n27p276

\section{Abstract}

This study estimates the technical efficiency of a sample of 411 maïzicoles farms in the main production areas in Benin. The study proposed an evaluation of the technical performance of these farms and identified factors of inefficiency by using the model of the production stochastic frontier Cobb-Douglas to assess the levels of efficiencies of systems production involved in the first step. A truncated regression was used in the second stage in order to establish a relationship between the indices of technical efficiency and some exogenous variables or attributes. The results of the first step shows that the index of technical efficiency varies from 37.37 to $96.22 \%$ for all systems, and that of the average technical efficiency is $80.35 \%$. The maximum value of technical efficiency of at least $92.75 \%$ obtained shows that there are very efficient producers in the study sample as being close to the production frontier. These producers who obtain such a high productivity can be referred to generally improve the productivity of maize production systems in Benin. The results of the second stage indicate that the variables such as access to fertilizers and herbicides, the use of 
animal traction and tractor, technical guidance and access to credit influence significantly $(\mathrm{p}<0.05$ to $\mathrm{p}<0.001)$ the technical efficiency of maize farms surveyed.

Keywords: Technical efficiency, stochastic frontier, efficiency index, maize, Benin

\section{Résumé}

Cette étude estime 1'efficacité technique d'un échantillon de 411 exploitations maïzicoles dans les principales zones de production au Bénin. L'étude a proposé une évaluation des performances techniques de ces exploitations et en a identifié les facteurs de l'inefficacité en utilisant le modèle de la frontière stochastique de production de type Cobb-Douglas en vue d'évaluer les niveaux d'efficacités des systèmes de production concernés dans la première étape. Une régression tronquée a été utilisée dans la deuxième étape en vue d'établir une relation entre les indices d'efficacité technique et quelques variables exogènes ou attributs. Les résultats de la première étape montrent que l'indice d'efficacité technique varie de 37,37 à $96,22 \%$ pour tous les systèmes, et celui de l'efficacité technique moyenne en est de 80,35\%. La valeur maximale d'efficacité technique d'au moins $92,75 \%$ obtenue, montre qu'il existe des producteurs très performants dans l'échantillon d'étude car étant proches de la frontière de production. Ces producteurs qui obtiennent une telle productivité élevée peuvent servir de référence pour améliorer de façon générale, la productivité des systèmes de production à base de maïs au Bénin. Les résultats de la deuxième étape indiquent que les variables telles que l'accès aux engrais et aux herbicides, l'utilisation de la traction animale et du tracteur, l'encadrement technique et l'accès au crédit influencent significativement $(p<0,05$ à $p<0,001)$ l'efficacité technique des exploitations de maïs enquêtées.

Mots-clés : Efficience technique, frontière stochastique, indice d'efficacités, maïs, Bénin

\section{Introduction}

Au Bénin, la production agricole est fluctuante d'une année à l'autre du fait des conditions climatiques, de la qualité des intrants agricoles, de la non maîtrise de l'eau, etc. Dans le même temps, la production de certaines denrées alimentaires telles que les céréales est tributaire des saisons de pluies alors que la consommation est quotidienne, régulière et croissante.

Dans la zone cotonnière du Nord-Bénin, selon Biaou et Gnimadi (2012), l'exploitation forestière contribue de plus en plus, à l'accélération du phénomène des dérèglements climatiques, agissant sur les rendements des 
cultures de maïs (Zea mays L.), aliment de base des populations au Bénin (Adégbola et al., 2013), et obligeant les producteurs à rechercher les moyens d'adaptation. En effet, le maïs est la seule céréale pour laquelle le Bénin dégage des excédents exportables vers les pays voisins, notamment le Niger et le Nigeria (Ahouannenou et Houénou, 2011).

Face à la montée des problèmes environnementaux et la dégradation des ressources naturelles, les gains de productivité en agriculture et l'efficience de l'utilisation des facteurs de production deviennent de plus en plus difficiles à réaliser. Dans cette perspective, l'Association pour la Relance Agronomique en Alsace [ARAA] (2009) estime que, adapter les systèmes de grande culture à la marge ne paraît pas suffisant pour répondre à leur contribution à la production, aux modèles de consommation et aux échanges extérieurs. L'agriculture d'aujourd'hui se trouve donc devant le défi de devoir améliorer la productivité des facteurs autres que le travail, tels que les consommations intermédiaires et le capital (Butault, 2006).

Depuis les années 1990 des efforts de développement économique ont été entrepris par les gouvernements successifs du Bénin ; cependant, le pays reste frappé par une pauvreté qui touche environ deux personnes sur cinq au niveau national (PNUD, 2011; BCEAO, 2012). En 2010, son Indicateur composite de Développement Humain (IDH) est de 0,435. Cet indice se situe sur une tendance à la hausse grâce aux efforts accomplis dans l'éducation et la santé depuis 1975 où il était estimé à 0,312 . En dépit de ces progrès, la pauvreté monétaire touche encore une partie importante de la population et n'a cessé d'augmenter ces dernières années. De ce fait, le Bénin est encore classé au $134^{\text {ème }}$ rang sur 169 pays au niveau mondial en matière d'IDH (INSAE, 2011 ; PNUD, 2011 ; BCEAO, 2012).

Pour résoudre leurs problèmes alimentaires et de revenu financier, certains petits producteurs s'impliquent dans la production de maïs, car cette culture est devenue une culture de rente, et est adaptée à toutes les zones agroécologiques du Bénin. Dans ces exploitations il est pratiqué différents systèmes de cultures et de productions. Dans une perspective d'optimisation de l'exploitation maïzicole, il est donc nécessaire d'évaluer parmi les différents systèmes adoptés, ceux qui sont techniquement plus efficaces. Dans une économie où la disponibilité des ressources est limitée, la notion d'efficacité technique prend une place de plus en plus importante dans les débats et les recherches scientifiques. Grâce à une recherche participative, on peut imaginer de nouveaux systèmes de culture de maïs plus durables, répondant à la fois aux exigences environnementales (réclamées par la société), aux exigences économiques (les exploitations agricoles doivent rester rentables), et aux exigences sociales (favorables aux conditions de vie des moins favorisés de la collectivité) en vue de leur contribution à l'amélioration de la sécurité alimentaire et aux revenus des agriculteurs. Il 
faut donc anticiper sur de futurs besoins et acquérir des données sur les systèmes de production innovants. Pour y parvenir, il convient entre autres de comprendre et d'analyser les relations qui s'établissent entre les systèmes de production de maïs pratiqués et l'ensemble englobant du système d'activités, tant du point de vue de la répartition de la force de travail (concurrentielle ou complémentaire) que de celui de l'accès et de l'utilisation du capital (humain, financier ou matériel). Cela permettra de mieux appréhender leurs niveaux d'efficacités en vue d'en dégager les meilleurs.

Selon Ghali et al. (2014), l'efficacité technique (TE) ou productive concerne la capacité de l'exploitation à éviter le gaspillage par une bonne gestion des ressources disponibles. Elle se décompose en Efficacité d'Échelle (EE) et en Efficacité Technique Pure (ETP). L'efficacité d'échelle renseigne sur le niveau optimal de la taille de l'exploitation; les exploitations qui opèrent avec un rendement d'échelle adéquat ont une élasticité d'échelle égale à 1 . L'efficacité technique pure reflète, quant à elle, la capacité d'une exploitation à atteindre une production maximale pour un ensemble d'intrants indépendamment des prix des facteurs et des produits. Elle renseigne sur les pratiques de gestion et d'organisation de l'unité de production (Latruffe, 2010 ; Blancard et al., 2013).

Le but de la présente recherche est de repérer les systèmes ayant les indices d'efficacités les plus élevés parmi l'ensemble des systèmes mis en comparaison, et qui offrent à la fois un cadre pour intégrer et interpréter la mesure de performances techniques des systèmes de production durables.

\section{Méthodologie}

\section{Localisation de l'espace de recherche et échantillonnage}

Le champ d'investigation s'étend sur 3 zones agroécologiques du Bénin, choisies de façon aléatoire dans les localités apparaissant comme régions les plus favorables à la production du maïs. 
Figure 1. Matérialisation de l'espace enquêté au Bénin.

Source : Données de l'enquête, octobre 2015.

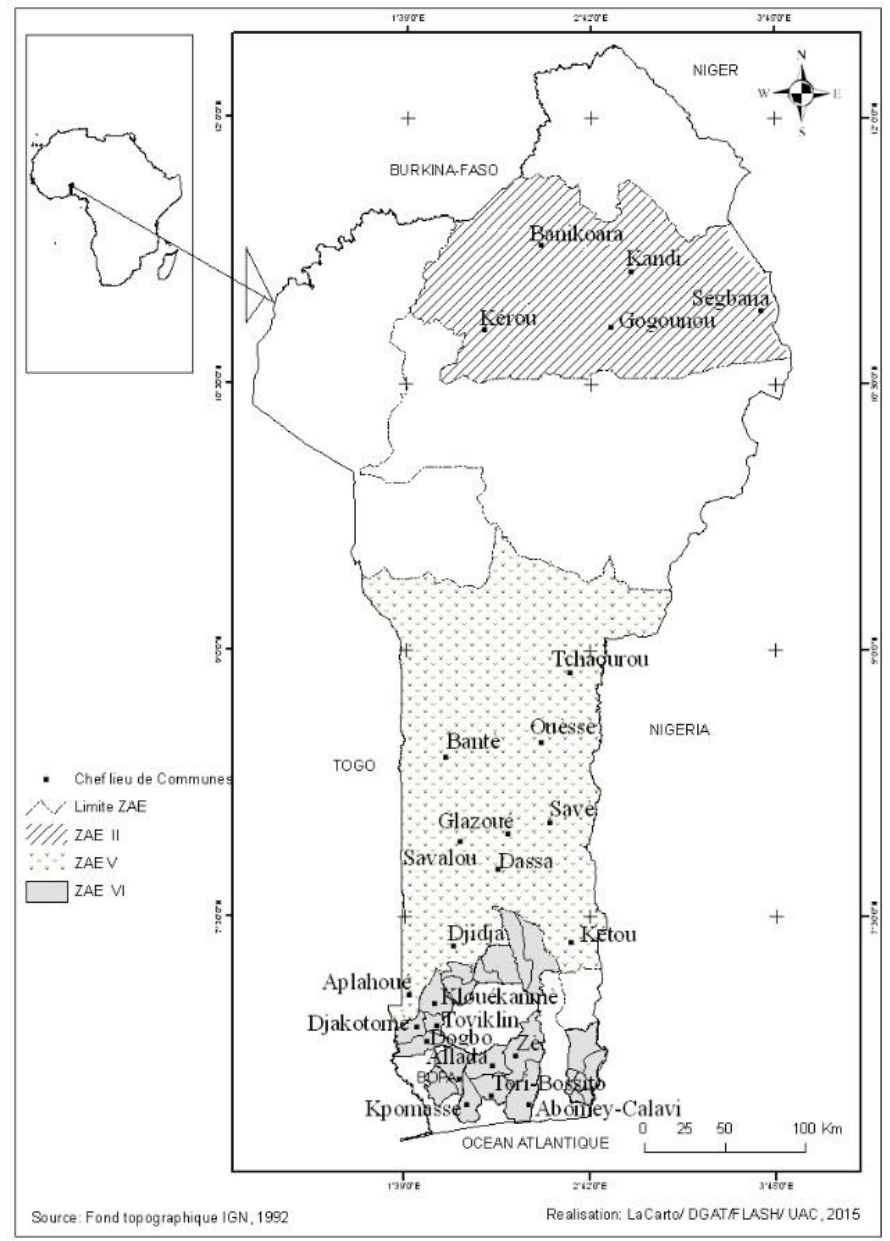

A partir d'une base de sondage de 631 exploitants de maïs échantillonnés dans 11 départements du Bénin, des analyses ont été réalisées sur 411 exploitants maïzicoles répartis comme suit : 49 exploitations dans la zone de recherche une, 115 au niveau de la zone deux et 247 dans la zone trois. Au total, 38 villages sont sélectionnés au sein de 38 communes dans les 11 départements. Les échantillons concernent les exploitants ayant produit du maïs au cours de la campagne agricole 2012-2013, et proviennent de la base de données du sous-projet CNS-Maïs/2012/05, portant sur l'évaluation agronomique et socio-économique des systèmes de production à base de maïs dans les zones agroécologiques du Bénin. Le mode de sélection des villages de recherche et des unités d'enquête adopté par le CNS-Maïs a été fait en trois étapes à partir de la formule à tirage stratifié non exhaustif de Neyman (1934) dans les 8 zones agroécologiques basées sur le découpage 
effectué par l'Institut National des Recherches Agricoles du Bénin (INRAB). Les trois étapes suivies pour l'échantillonnage sont les suivantes : 1) Effectif global des ménages selon la base de données de l'INSAE ; 2) Extraction de l'effectif réduit maïsiculteurs à partir de l'échantillon des ménages agricoles ; et 3) Rétention de l'échantillon final des maïsiculteurs à partir de $\mathrm{n} \geq 6$.

La formule de calcul des effectifs se présente comme suit :

$\mathbf{n}$ : la taille de l'échantillon,

$$
n=t^{2} \cdot \frac{p \cdot(1-p)}{e^{2}} \cdot \frac{N-n^{\prime}}{N-1}
$$

$\mathbf{N}$ : la taille des ménages, $\mathbf{n}$ : l'effectif des ménages agricoles.

e : la marge d'erreur. Elle est fixée à $10 \%$ qui est une norme internationalement reconnue. Ceci voudra dire que le choix de l'échantillon est acceptable dans un intervalle de confiance de $90 \%$.

$\mathbf{t}$ : le coefficient de marge ( $\mathrm{T}$ de student) déduit du taux de confiance. Cette statistique qui avoisine 1 permet de dire que le seuil de signification est fixe à $10 \%$.

p : la proportion des ménages agricoles dans l'effectif total des ménages dans chaque commune.

\section{Les modèles d'estimation des indices d'efficacité technique}

L'équation de la frontière de production stochastique

Le classement de la frontière de production en fonction de la forme de frontière, permet de distinguer les approches paramétriques et non paramétriques. La nature des écarts entre la production observée et la production maximale différencie les frontières stochastiques des frontières déterministes. En effet, si l'on suppose que les écarts sont expliqués uniquement par l'inefficacité du producteur, on qualifie la frontière de nature déterministe ; si par contre on estime que les écarts sont expliqués à la fois par l'inefficacité du producteur et par des éléments aléatoires qui ne dépendent pas du producteur, on dit que la frontière est de nature stochastique. Etant donné le caractère aléatoire de la production de maïs au Bénin, lié à la nature du climat et aux fluctuations de prix des intrants et des produits agricoles, le choix de l'approche stochastique pour estimer l'efficacité technique nous paraît justifié pour calculer les estimations. La frontière de production stochastique par la fonction Cobb-Douglas est définie par :

$\ln \left(Q_{i}\right)=\beta_{0}+\beta_{1} \ln \left(K_{i}\right)+\beta_{2} \ln \left(L_{i}\right)+v_{i}-u_{i}$, où $Q_{i}$ représente l'output, $K_{i}$ et $L_{i}$ sont des inputs respectivement le capital et le travail, avec $v_{i}$ et $u_{i}$ les variables aléatoires distribuées selon une loi normale d'espérance nulle, et comme des variables aléatoires non négatives, et $i$ les observations de 1,2, ..., N (Coelli, 1996). 
Une mesure de l'efficacité technique est le ratio de la production minimale sur la production observée ; le modèle d'estimation des scores de l'efficacité technique se présente comme suit :

$E P_{i}=\frac{P_{i}^{\min }}{P_{i}}=\frac{P\left(X_{i}, P_{i}, \propto\right) e^{V_{i}}}{P\left(X_{i}, P_{i}, \propto\right) e^{V_{i}+U_{i}}}=e^{-U_{i}}, \quad$ avec $\quad 0 \leq E P_{i}^{*} \leq 1 ; P_{i}^{\text {min }}$ étant $\quad$ la production minimale de l'observation $i$.

Spécification empirique de la frontière stochastique

En utilisant l'approche paramétrique pour la détermination des indices d'efficacité technique, une frontière de production stochastique de type Cobb-Douglas est estimée. Les paramètres de la frontière de production stochastique ont été estimés par la méthode du maximum de vraisemblance. Mathématiquement, si l'on considère un maïsiculteur qui combine des facteurs de production (superficie, main-d'œuvre, capital) pour produire un bien QPROD (le maïs), la frontière de production stochastique est représentée par la formule suivante :

$\ln \left(Q P R O D_{i}\right)=\beta_{0}+\beta_{1} \ln \left(Q S E M_{i}\right)+\beta_{2} \ln \left(Q E N G R A I_{i}\right)+$ $\beta_{3} \ln \left(Q H E R B I_{i}\right)+\beta_{4} \ln \left(Q M O F_{i}\right)+\beta_{5} \ln \left(Q M O S_{i}\right)+\beta_{6} \ln \left(Q M A C H I N_{i}\right)+$ $\beta_{7} \ln \left(M A T E_{i}\right)+\beta_{8} \ln \left(S U P_{i}\right)+v_{i}-u_{i}$, où $\boldsymbol{L n}$ représente le logarithme népérien et $\boldsymbol{i}$ le producteur; $\boldsymbol{v}$ les variables aléatoires hors du contrôle des producteurs, elles sont supposées être indépendamment et identiquement distribuées selon une loi normale d'espérance mathématique nulle et de variance $\sigma_{v}^{2}\left[V_{i} \approx N\left(0, \sigma_{v}^{2}\right)\right]$, indépendantes des $u$. $\boldsymbol{u}$ : représente les variables aléatoires d'inefficacité technique et sont supposées être indépendamment et identiquement distribuées comme des variables aléatoires non négatives obtenues par une troncature à zéro, de la distribution de type $N\left(\mu, \sigma_{u}^{2}\right)$. Les $\beta, \mu$ et $\sigma^{2}$ sont les paramètres à estimer par la méthode du maximum de vraisemblance (MV) au niveau du modèle, à l'aide du programme FRONTIER 4.1 de Coelli (1996). Ces paramètres sont les coefficients de la frontière de production dont les résidus permettront de déterminer les indices d'efficacité technique, et plus précisément par la formule suivante définie par Coelli et al. (1998): $T E_{i}=\exp \left(-u_{i}\right)$.

Les variables incluses dans le modèle sont définies comme suit : QPROD : la production totale de maïs $(\mathrm{kg})$ au cours de la campagne considérée; $\boldsymbol{Q S E M}$ : la quantité totale de semence utilisée $(\mathrm{kg})$; QENGRAI : la quantité totale d'engrais NPK et Urée utilisée $(\mathrm{kg})$; $\boldsymbol{Q H E R B I}$ : la quantité totale d'herbicide utilisée $(\mathrm{kg}) ; \boldsymbol{Q M O F}$ : la quantité totale de main-d'œuvre familiale utilisée est évaluée en homme-jour (h.j), elle regroupe la main-d'œuvre familiale et la main-d'œuvre d'entraide ; cet input est exprimé en nombre de travailleurs par jour. $Q \boldsymbol{M O}$ : la quantité de main-d'œuvre salariée utilisée (h.j) ; cet input est exprimé en nombre de travailleurs salariés par jour. QMACHIN : la quantité totale en équipements loués (capital fixe), dans la production du maïs, évaluée en valeur totale des 
annuités ; MATE : la quantité de petits matériels utilisés dans la production du maïs, évaluée en valeur totale des annuités; SUP : Superficie totale emblavée (ha) en maïs par l'exploitant au cours de la campagne agricole considérée.

Le modèle a été estimé par la méthode des Moindres Carrés Ordinaires (MCO). De plus, des tests de Durbin-Waston, de Goldfeld et Quandt et de Farrar-Glauber ont été réalisés afin de vérifier respectivement si des erreurs d'autocorrélation, d'hétérocédasticité et de multicolinéarité existent et sont significatives. En particulier, les erreurs de multicolinéarité ont été corrigées par la méthode d'estimation par étapes qui élimine au fur et à mesure les variables fortement corrélées à d'autres dans le modèle jusqu'à l'obtention d'une estimation sans erreur de multicolinéarité.

\section{Déterminants de l'efficacité technique}

L'analyse des déterminants des différentes efficacités amène à établir une relation entre les indices d'efficacité technique et les variables ou attributs socio-économiques, démographiques et communautaires. À cet effet, les scores d'efficacités obtenus pour chaque type d'exploitation sont régressés sur les déterminants potentiels. Ce sont la taille des superficies, les rendements obtenus, l'utilisation des mains-d'œuvre (salariée, familiale, entraide), l'utilisation ou non d'engrais minéraux, l'utilisation ou non de la traction animale et du tracteur et l'utilisation ou non des variétés améliorées. La première question habituellement posée est comment expliquer le différentiel d'efficacité aperçu entre les exploitations et/ou entre les zones ? Pour y parvenir, la méthode utilisée pour la régression est la méthode d'estimation Tobit, étant donné le caractère censuré à 0 des variables expliquées (Maddala, 1983).

Le modèle Tobit se réfère aux modèles à variable dépendante limitée pour lesquels la variable dépendante est continue mais observable seulement sur un intervalle spécifique. En d'autres termes, le domaine de la variable dépendante est contraint à un espace limité par les observations possibles. Les modèles à variable dépendante découlent des modèles à variables qualitatives, qu'on utilise lorsqu'on désire évaluer la probabilité que la variable dépendante appartienne à l'intervalle pour lequel elle est observable. Pour ce faire, les scores d'efficacités déterminés pour chaque type d'exploitation sont régressés sur les déterminants potentiels.

\section{Les outils de base}

Une base de données de tous les systèmes répertoriés a été constituée grâce au logiciel Excel 2013. Par la suite, les analyses statistiques et 
économétriques ont été effectuées à l'aide du programme SPSS 16.0. L'approche paramétrique a été adoptée pour estimer la frontière de production des exploitations agricoles échantillonnées. Les paramètres des frontières de chaque système de production sont alors estimés à partir des données de l'échantillon par une méthode du maximum de vraisemblance (MV), à l'aide du programme FRONTIER 4.1 de Coelli (1996).

\section{Résultats}

\section{Analyse du niveau d'efficacité technique des systèmes de production} exploitants

Caractéristiques démographiques et socioéconomiques des

Les informations traitées dans la présente sous-section permettent non seulement de déterminer les niveaux d'efficacités des systèmes de production des exploitations étudiées, mais surtout de caractériser les systèmes performants.

Quatre principaux systèmes de production à base de maïs au Bénin ont été caractérisés. Ils sont essentiellement fondés sur la taille des superficies, les rendements, les coûts des mains-d'œuvre, les proportions d'utilisation de la traction animale et du tracteur, les coûts d'utilisation des engrais minéraux et des herbicides, et les techniques et stratégies culturales. Il s'agit des types ci-après :

- Type 1: Grandes exploitations à production intensive, souvent en faire-valoir direct sur monoculture. Les promoteurs font de gros investissements sur des superficies allant de 6 à 22 ha dans un système utilisant indifféremment tracteur et traction animale, avec suffisamment d'engrais minéraux et d'herbicides et une forte main-d'œuvre pour une production de maïs destinée au marché.

Type 2: Exploitations moyennes. Système intermédiaire entre les 4 systèmes identifiés, celui-ci utilise indifféremment et moyennement le tracteur et l'attelage, la main-d'œuvre salariée sur des superficies variant entre 2 et 12,60 ha. La production de maïs de ces exploitations est destinée autant à la consommation qu'au marché.

Type 3 : Petites exploitations de subsistance peu productives, optant pour la sécurité alimentaire de l'exploitant. Ce type s'identifie à un système de polyculture vivrière appliquée à des petites parcelles ou exploitations ayant de très petites superficies, à travaux essentiellement manuels avec peu d'investissement sur des étendues oscillant majoritairement autour de 0,30 ha, et rarement 6 ha. Ces exploitations ont les plus faibles rendements et leur production est destinée essentiellement à la subsistance.

Type 4 : Très grandes exploitations à production extensive à gros investissements. Ce type régit les exploitations de 29 à 60 hectares. Ces exploitations n'utilisent pas du tout le tracteur, mais leurs exploitants 
investissent essentiellement dans la traction animale, utilisent abondamment les engrais minéraux et les herbicides, et moyennement la main-d'œuvre. Leur système maïzicole repose sur un modèle de production itinérante, alterné au cotonnier. Ils visent prioritairement la maïsiculture de rente.

Le tableau 1 présente les résultats de l'analyse descriptive des variables démographiques et socio-économiques des exploitants suivis selon les 4 types de systèmes de production au Bénin, et nous inspire les commentaires suivants : Comptant pour 94,2\% de l'échantillon étudié, les hommes représentent la grande majorité des producteurs de l'ensemble des 4 types de systèmes de production, et sont représentés par une majorité absolue dans la classe 4 nommée système de "Très grandes exploitations à production extensive". Ce type de système, rassemblant les utilisateurs de la traction animale, ne peut réunir essentiellement que des hommes en raison de la difficulté de l'entretien des bêtes et de la délicatesse de la manipulation de ce modèle de gestion.

Tableau 1. Caractéristiques démographiques et socio-économiques des exploitations en fonction du type de système de production.

\begin{tabular}{|c|c|c|c|c|c|c|}
\hline \multirow[b]{2}{*}{ Variables } & \multirow[b]{2}{*}{ Modalités } & \multicolumn{4}{|c|}{ Proportion des répondants $(\%)$} & \multirow[b]{2}{*}{$\begin{array}{c}\text { Ensemble } \\
(n=411)\end{array}$} \\
\hline & & $\begin{array}{c}\text { Grandes } \\
\text { exploitations } \\
\text { à production } \\
\text { intensive } \\
(\mathrm{n}=51)\end{array}$ & $\begin{array}{c}\text { Exploitations } \\
\text { moyennes } \\
(\mathrm{n}=121)\end{array}$ & $\begin{array}{c}\text { Petites } \\
\text { exploitations } \\
\text { de } \\
\text { subsistance } \\
(\mathrm{n}=230)\end{array}$ & $\begin{array}{l}\text { Très grandes } \\
\text { exploitations } \\
\text { à production } \\
\text { extensive } \\
(\mathrm{n}=09)\end{array}$ & \\
\hline \multirow{2}{*}{ Sexe } & Féminin & 3,9 & 1,7 & 8,7 & 0,0 & 5,8 \\
\hline & Masculin & 96,1 & 98,3 & 91,3 & 100,0 & 94,2 \\
\hline \multirow{3}{*}{ Age } & $\leq 30$ ans & 7,8 & 12,4 & 10,9 & 0,0 & 10,7 \\
\hline & $\begin{array}{c}\text { Entre } 31 \text { et } 60 \\
\text { ans }\end{array}$ & 86,3 & 72,7 & 80,9 & 88,9 & 79,3 \\
\hline & $\geq 61$ ans & 5,9 & 14,9 & 8,3 & 11,1 & 10,0 \\
\hline \multirow{2}{*}{ Alphabétisé } & Non & 45,1 & 58,7 & 68,3 & 66,7 & 62,5 \\
\hline & Oui & 54,9 & 41,3 & 31,7 & 33,3 & 37,5 \\
\hline \multirow{4}{*}{ Instruit } & Non & 19,6 & 43,0 & 53,0 & 55,6 & 46,0 \\
\hline & $\begin{array}{l}\text { Niveau } \\
\text { primaire }\end{array}$ & 62,7 & 47,1 & 34,3 & 44,4 & 41,8 \\
\hline & $\begin{array}{l}\text { Niveau } \\
\text { collège }\end{array}$ & 15,7 & 7,4 & 9,6 & 0,0 & 9,5 \\
\hline & $\begin{array}{l}\text { Niveau } \\
\text { supérieur }\end{array}$ & 2,0 & 2,5 & 3,0 & 0,0 & 2,7 \\
\hline \multirow{3}{*}{$\begin{array}{l}\text { Fréquence } \\
\text { d'appui } \\
\text { technique }\end{array}$} & 1 fois l'an & 51,0 & 72,7 & 79,1 & 33,3 & 72,7 \\
\hline & $\begin{array}{l}1 \text { fois par } \\
\text { mois }\end{array}$ & 39,2 & 19,8 & 16,1 & 55,6 & 20,9 \\
\hline & $\begin{array}{l}2 \text { fois par } \\
\text { mois }\end{array}$ & 3,9 & 3,3 & 1,7 & 11,1 & 2,7 \\
\hline
\end{tabular}




\begin{tabular}{ccccccc}
\hline $\begin{array}{c}3 \text { fois par } \\
\text { mois } \\
1 \text { fois par } \\
\text { semaine }\end{array}$ & 0,0 & 1,7 & 1,3 & 0,0 & 1,2 \\
& 5,9 & 2,5 & 1,7 & 0,0 & 2,4 \\
\hline $\begin{array}{c}\leq 6 \text { ha } \\
>6 \text { et } \leq 12,60 \\
\text { ha }\end{array}$ & 2,0 & 74,4 & 100,0 & 0,0 & 78,1 \\
Superficie & 56,9 & 25,6 & 0,0 & 0,0 & 14,6 \\
& $\begin{array}{c}12,60 \text { et } \leq \\
22 \text { ha }\end{array}$ & 41,2 & 0,0 & 0,0 & 0,0 & 5,1 \\
$>22$ ha & 0,0 & 0,0 & 0,0 & 100,0 & 2,2 \\
\hline
\end{tabular}

Source : Données de l'enquête (2013).

L'âge moyen de la plupart des chefs d'exploitation maïzicoles $(79,3 \%)$ se situe entre 31 et 60 ans, donc constitués d'adultes et ces exploitants se retrouvent par ailleurs en majorité $(88,9 \%)$ dans la classe des "Très grandes exploitations à production extensive", ainsi que dans celle des "Grandes exploitations à production intensive". En effet, ces modèles, requérant de la force pour l'entretien des bêtes et surtout pour la manipulation de la traction ou du tracteur, sont à juste titre conduits par les exploitants d'âge adulte. Ce même tableau montre que bon nombre de producteurs sont alphabétisés $(37,5 \%)$ à travers toutes les zones d'observation, et savent alors lire et écrire dans leur langue locale.

Mais le score le plus impressionnant se retrouve au niveau de la classe des "Grandes exploitations à production intensive" à près de $55 \%$, où les exploitants sont appelés à manipuler le tracteur ou la traction, donc avec pour exigence de savoir lire les notices ou noter les consignes techniques des inventeurs. Cela confirme la contrainte existant dans ce modèle de gestion. On peut déduire de ces observations que les niveaux d'alphabétisation ou d'instruction sont autant de variables qui peuvent influencer la prise de décision dans la gestion de l'exploitation agricole et par conséquent sur l'efficacité de production, du fait que les exploitations comprenant plus de personnes instruites ou alphabétisées se comportent mieux. De cette analyse statistique, on retient également que les petits exploitants reçoivent faiblement l'encadrement technique; en effet, ils reçoivent la visite de l'encadrement en moyenne une fois l'an, pour les $79,1 \%$ d'entre eux. Les exploitants les mieux encadrés sont les plus nantis notamment ceux des première et quatrième classes avec respectivement une visite par semaine pour la classe 1 "Grandes exploitations à production intensive" à 5,9\% de son effectif, et une visite le mois à $39,2 \%$ pour cette même classe et à $55,6 \%$ pour la classe 4 "Très grandes exploitations à production extensive". Cette analyse descriptive prouve enfin, que les superficies sont strictement petites et très grandes pour $100 \%$ de leurs effectifs respectivement pour les classes 3 
"Petites exploitations de subsistance", et 4 "Très grandes exploitations à production extensive" ; ce qui confirme leurs dénominations.

\section{Frontière de production stochastique}

Les indices d'efficacité technique sont calculés en se fondant sur la typologie ci-dessus des exploitations de maïs dans la zone d'étude. Pour estimer le modèle de frontière de production de type Cobb-Douglas, les variables suivantes ont été testées : la production totale de maïs $(\mathrm{kg})$, la quantité totale de semence utilisée $(\mathrm{kg})$, la quantité totale d'engrais (NPK et Urée) utilisé $(\mathrm{kg})$, la quantité totale d'herbicide utilisé $(\mathrm{kg})$, la quantité totale de main-d'œuvre utilisée (h.j), la valeur totale des annuités des équipements (capital fixe) utilisés dans la production du maïs.

Les résultats du modèle de régression estimé sont présentés dans le tableau 2.

Tableau 2. Résultats de la fonction de production frontière de type Cobb-Douglas.

\begin{tabular}{|c|c|c|c|c|c|c|}
\hline \multirow[b]{2}{*}{ Variables } & \multirow[b]{2}{*}{$\begin{array}{c}\text { Coefficient } \\
\text { s }\end{array}$} & \multicolumn{4}{|c|}{ Systèmes de production } & \multirow[b]{2}{*}{$\begin{array}{c}\text { Ensembl } \\
\mathrm{e}\end{array}$} \\
\hline & & $\begin{array}{c}\text { Grandes } \\
\text { exploitation } \\
\text { s à } \\
\text { production } \\
\text { intensive }\end{array}$ & $\begin{array}{c}\text { Exploitation } \\
\text { s moyennes }\end{array}$ & $\begin{array}{c}\text { Petites } \\
\text { exploitation } \\
\text { s de } \\
\text { subsistance }\end{array}$ & $\begin{array}{c}\text { Très grandes } \\
\text { exploitation } \\
\text { s à } \\
\text { production } \\
\text { extensive }\end{array}$ & \\
\hline \multicolumn{7}{|c|}{ Frontière stochastique de production } \\
\hline Constante & $\beta_{0}$ & $\begin{array}{c}1,94 \\
(1,73)\end{array}$ & $\begin{array}{c}12,16 * * * \\
(1,12)\end{array}$ & $\begin{array}{c}3,90 * * * \\
(0,27)\end{array}$ & - & $\begin{array}{c}3,95 * * * \\
(0,21)\end{array}$ \\
\hline LnQSEM & $\beta_{1}$ & $\begin{array}{l}0,76 * * \\
(0,27)\end{array}$ & $\begin{array}{c}-1,75^{* * * *} \\
(0,31)\end{array}$ & $\begin{array}{c}0,85^{* * * *} \\
(0,12)\end{array}$ & - & $\begin{array}{c}0,83 * * * \\
(0,09)\end{array}$ \\
\hline LnQENGRAI & $\beta_{2}$ & $\begin{array}{c}0,84 * * * \\
(0,07)\end{array}$ & $\begin{array}{l}0,15^{* *} \\
(0,03)\end{array}$ & $\begin{array}{c}0,10^{* * *} \\
(0,01)\end{array}$ & - & $\begin{array}{c}0,12 * * * \\
(0,01)\end{array}$ \\
\hline LnQHERBI & $\beta_{3}$ & $\begin{array}{c}0,04 * * * \\
(0,06)\end{array}$ & $\begin{array}{c}0,03 \\
(0,03)\end{array}$ & - & - & $\begin{array}{c}0,01 \\
(0,02)\end{array}$ \\
\hline LnQMOF & $\beta_{4}$ & $\begin{array}{l}-0,10 \\
(0,03)\end{array}$ & $\begin{array}{c}0,06 \\
(0,04)\end{array}$ & $\begin{array}{l}-0,09 \\
(0,07)\end{array}$ & - & $\begin{array}{l}-0,02 \\
(0,03)\end{array}$ \\
\hline LnQMOS & $\beta_{5}$ & $\begin{array}{l}-0,14^{*} \\
(0,05)\end{array}$ & $\begin{array}{c}0,01 \\
(0,07)\end{array}$ & $\begin{array}{c}0,06 \\
(0,07)\end{array}$ & - & $\begin{array}{c}0,01 \\
(0,04)\end{array}$ \\
\hline LnMACHIN & $\beta_{6}$ & $\begin{array}{c}0,05^{* * *} \\
(0,01)\end{array}$ & $\begin{array}{l}0,07 * \\
(0,02)\end{array}$ & $\begin{array}{l}0,07 * * \\
(0,02)\end{array}$ & - & $\begin{array}{c}0,04 * * \\
(0,01)\end{array}$ \\
\hline LnMATE & $\beta_{7}$ & $\begin{array}{c}-0,30 * * \\
(0,08)\end{array}$ & $\begin{array}{l}-0,14 \\
(0,08)\end{array}$ & $\begin{array}{c}0,00 \\
(0,06)\end{array}$ & - & $\begin{array}{l}-0,03 \\
(0,05)\end{array}$ \\
\hline LnSup & $\beta_{8}$ & $\begin{array}{l}-0,31 \\
(0,52)\end{array}$ & $\begin{array}{c}2,66 * * * \\
(0,34)\end{array}$ & $\begin{array}{l}0,20 * \\
(0,08)\end{array}$ & - & $\begin{array}{l}0,21^{*} \\
(0,07)\end{array}$ \\
\hline \multicolumn{7}{|c|}{ Paramètres d'efficience } \\
\hline Sigma-carré & $\sigma^{2}$ & $\begin{array}{l}0,05^{*} \\
(0,02)\end{array}$ & $\begin{array}{c}0,08 * * * \\
(0,01)\end{array}$ & $\begin{array}{l}0,21 * \\
(0,07)\end{array}$ & - & $\begin{array}{c}0,15 * * * \\
(0,03)\end{array}$ \\
\hline Gamma & $\gamma$ & $\begin{array}{c}1,00^{* * * *} \\
(0,00)\end{array}$ & $\begin{array}{c}1,00^{* * * *} \\
(0,00)\end{array}$ & $\begin{array}{c}0,83^{* * * *} \\
(0,07)\end{array}$ & - & $\begin{array}{c}0,89 * * * \\
(0,03)\end{array}$ \\
\hline
\end{tabular}


Log de

vraisemblanc

e

Test du ratio

de

vraisemblanc

e

Degré de liberté

46,03

29,58

LR

60,06

23,88

59,83

NB : les niveaux de signification sont : *** $: \mathrm{p}<0,001 ; *^{*}: \mathrm{p}<0,01 ; *: \mathrm{p}<0,05$. Les valeurs entre parenthèse sont les erreurs types,

Source : Données de l'enquête (2013),

Des résultats du tableau 2 nous pouvons retenir ce qui suit : pour l'ensemble des systèmes de production, la valeur de $\gamma$ indique qu'environ $89 \%$ de la variation de la production du maïs est due à l'inefficacité technique. Selon $\sigma^{2}, 15 \%$ seulement, de cette variabilité sont attribués aux facteurs aléatoires.

Du tableau 2 également, il ressort que l'estimation de la frontière stochastique pour le type 4 système des "Très grandes exploitations $\grave{a}$ production extensive" n'est pas appropriée aux observations de ce système. En effet, la taille de ce sous-échantillon est très faible $(<30)$ et sensiblement égale au nombre de paramètres estimés (08), la petitesse du ddl (degré de liberté) ${ }^{1}$ empêche du coup la régression du modèle, donc ne permet pas de prendre de décision. Le chercheur n'étant plus en position d'ajuster la taille des sous-échantillons, il n'a pas été possible d'estimer et d'interpréter la frontière du système concerné.

Toujours conformément au tableau 2, des huit variables explicatives de la fonction production pour l'ensemble des systèmes, il s'avère que seuls les coefficients de la quantité des semences utilisées, de la quantité d'engrais et de l'utilisation de la mécanisation et de la superficie emblavée sont positifs et statistiquement significatifs. Ainsi, le coefficient de la quantité des semences utilisées est de 0,83 . Cela suggère que lorsque la quantité de semence utilisée s'augmente de $10 \%$, la production de maïs augmente de $8,30 \%$. Le coefficient de la quantité d'engrais utilisé est de 0,12 . Il en découle que lorsque la quantité d'engrais augmente de $10 \%$, la production de maïs augmente de 1,20\%. Quant au coefficient de l'utilisation de la mécanisation agricole, il est de 0,04. Ainsi, une intensification de la mécanisation à $10 \%$ entraîne un accroissement de la production du maïs de $0,40 \%$. L'accroissement de l'étendue de la superficie emblavée étant de 0,21 , on peut en déduire que l'augmentation des superficies de $10 \%$ augmente la production de $2,10 \%$.

${ }^{1}$ Selon la loi $\mathrm{t}$ de Student, le nombre de ddl = n-k. Dans le cas d'espèce, le coefficient à estimer $9-8=1$. 
La plupart des coefficients de ces mêmes variables sont statistiquement significatifs pour les trois systèmes dont la fonction de production frontière est estimée à savoir les "Grandes exploitations à production intensive", les "Exploitations moyennes", et les "Petites exploitations de subsistance"; positifs chez deux d'entre eux (Grandes exploitations à production intensive, et Petites exploitations de subsistance). Par contre, il est remarqué que le coefficient de la variable "quantité de semences" est négatif et très significatif chez celui des "Exploitations moyennes"; cela suppose que dans les exploitations moyennes, il y a mauvaise utilisation de semences, surtout qu'ils sont dans leur majorité, utilisateurs de semences améliorées.

\section{Mesures d'indices d'efficacité technique}

Les indices d'efficacité technique ont été directement obtenus avec le programme FRONTIER 4.1 (Coelli, 1996). Les résultats du tableau 3 montrent que l'indice d'efficacité technique varie de 37 à $96 \%$ pour l'ensemble des systèmes, et celui de l'efficacité technique moyenne est aussi très élevé $(80,35 \%)$. En moyenne, on note une plus grande efficacité pour les "grandes exploitations" avec respectivement 84,32 et $80,85 \%$ comparativement aux 2 autres types.

Tableau 3. Niveau d'efficacité technique par système et pour l'ensemble des systèmes.

\begin{tabular}{cccccc}
\hline & \multicolumn{5}{c}{ Système de production } \\
\cline { 2 - 5 } Niveau \\
d'efficacité & $\begin{array}{c}\text { Grandes } \\
\text { exploitations à } \\
\text { production } \\
\text { intensive }\end{array}$ & $\begin{array}{c}\text { Exploitations } \\
\text { moyennes }\end{array}$ & $\begin{array}{c}\text { Petites } \\
\text { exploitations de } \\
\text { subsistance }\end{array}$ & $\begin{array}{c}\text { Très grandes } \\
\text { exploitations à } \\
\text { production } \\
\text { extensive }\end{array}$ & Ensemble \\
\hline $\begin{array}{c}\text { Moyen } \\
(\%)\end{array}$ & 84,32 & 80,74 & 79,24 & - & 80,35 \\
$\begin{array}{c}\text { Minimum } \\
(\%)\end{array}$ & 54,75 & 40,00 & 37,37 & - & 37,37 \\
$\begin{array}{c}\text { Maximum } \\
(\%)\end{array}$ & 92,75 & 94,79 & 96,22 & - & 96,22 \\
\hline
\end{tabular}

Source : Données de l'enquête (2013),

Ce résultat peut s'expliquer par une plus grande maîtrise de la production dans la première classe qui bénéficie en permanence des assistances techniques, avec des niveaux d'instruction plus élevés et de biens d'équipements plus performants. Le plus faible niveau d'efficacité technique est observé dans le type "Petites exploitations de subsistance" où l'utilisation de semences améliorées et d'engrais chimiques est moins importante sur des sols très pauvres, avec des outils aratoires rudimentaires et pas du tout d'herbicide. Cependant, le système des "Petites exploitations de subsistance" 
apparaît comme celui pour lequel les producteurs ont un niveau d'efficacité maximum plus élevé.

Une réflexion plus approfondie permet de voir que l'élévation de l'étendue des superficies peut affecter l'aptitude ou la capacité du producteur à respecter les paquets technologiques de production. Ces producteurs qui obtiennent une telle productivité élevée peuvent servir de référence pour améliorer de façon générale, la productivité des systèmes de production à base de maïs au Bénin.

A partir de la fonction de production stochastique de type CobbDouglas, les potentiels en réduction des quantités de facteurs de production de maïs ont été calculés sur la base de l'effet d'inefficacité technique dérivée tout en maintenant constant le niveau de production. Le tableau 4 présente ces potentiels en réduction des quantités de facteurs de production utilisés.

Tableau 4. Pourcentages de réduction potentielle de quantités d'intrants de production.

\begin{tabular}{cccccc}
\hline & \multicolumn{4}{c}{ Systèmes de production } & \\
\cline { 2 - 5 } $\begin{array}{c}\text { Niveau } \\
\text { d'efficacité }\end{array}$ & $\begin{array}{c}\text { Grandes } \\
\text { exploitations à } \\
\text { production } \\
\text { intensive }\end{array}$ & $\begin{array}{c}\text { Exploitations } \\
\text { moyennes }\end{array}$ & $\begin{array}{c}\text { Petites } \\
\text { exploitations de } \\
\text { subsistance }\end{array}$ & $\begin{array}{c}\text { Très grandes } \\
\text { exploitations à } \\
\text { production } \\
\text { extensive }\end{array}$ & Ensemble \\
\hline $\begin{array}{c}\text { Efficacité } \\
\text { moyenne }(\%) \\
\begin{array}{c}\text { Faible } \\
\text { efficacité } \\
(\%)\end{array}\end{array} \quad 9,09$ & 14,82 & 17,65 & - & 16,49 \\
\hline
\end{tabular}

Source : Données de l'enquête (2013).

Se fondant sur les tableaux 3 et 4 , on peut espérer que si le producteur moyen de tous les systèmes de production à base de maïs devrait atteindre la performance des producteurs les plus performants, il pourrait réaliser une économie de ressources de 16,49\% [1-(80,35/96,22)] sur ses dépenses actuelles de production. De même, si le producteur qui est techniquement moins efficace devrait atteindre l'efficacité technique des meilleurs producteurs de la région, il pourrait épargner 61,16\% [1$(37,37 / 96,22)]$ de ses dépenses en ressources productives. Le cas particulier du système de production des "Grandes exploitations à production intensive" interpelle le chercheur sur les contre-performances observées même dans les potentiels de gains sur les quantités de facteurs de production pour ce type de système. En effet, son efficacité moyenne est de 84,32 (donc plus élevée), pour l'intervalle variant de 54,75 à $92,75 \%$. Le producteur moyen pourrait donc réaliser un gain de $9,09 \%$ sur ses dépenses de production dans la classe 1. Et si le producteur le moins efficace devrait atteindre l'efficacité du producteur le plus performant, il réaliserait un gain de $40,97 \%$ de ses dépenses en facteurs de production pour la même classe. La faiblesse de 
l'écart ainsi constaté par rapport aux deux systèmes moyens $\left(2^{\text {ème }}\right.$ et $3^{\text {ème }}$ classes) est justifiée car elle démontre le faible écart de gain perdu et qui reste à combler au niveau de leurs différents exploitants dans la mesure où ces types de producteurs n'ont pas perdu grand-chose comparativement à ceux des classes moyennes.

\section{Distribution de fréquences des indices d'Efficacité Technique}

La figure 3 présente la distribution de fréquences des indices d'efficacité technique pour les 3 systèmes.

Cette distribution montre pour l'ensemble des systèmes de production, que la plus grande majorité des exploitants $(39,1$ à $74,5 \%)$ opèrent entre 81 et $90 \%$ d'efficacité technique.

Figure 2. Distribution de fréquences des indices d'efficacité technique.

Source : Données de l'enquête (2013).
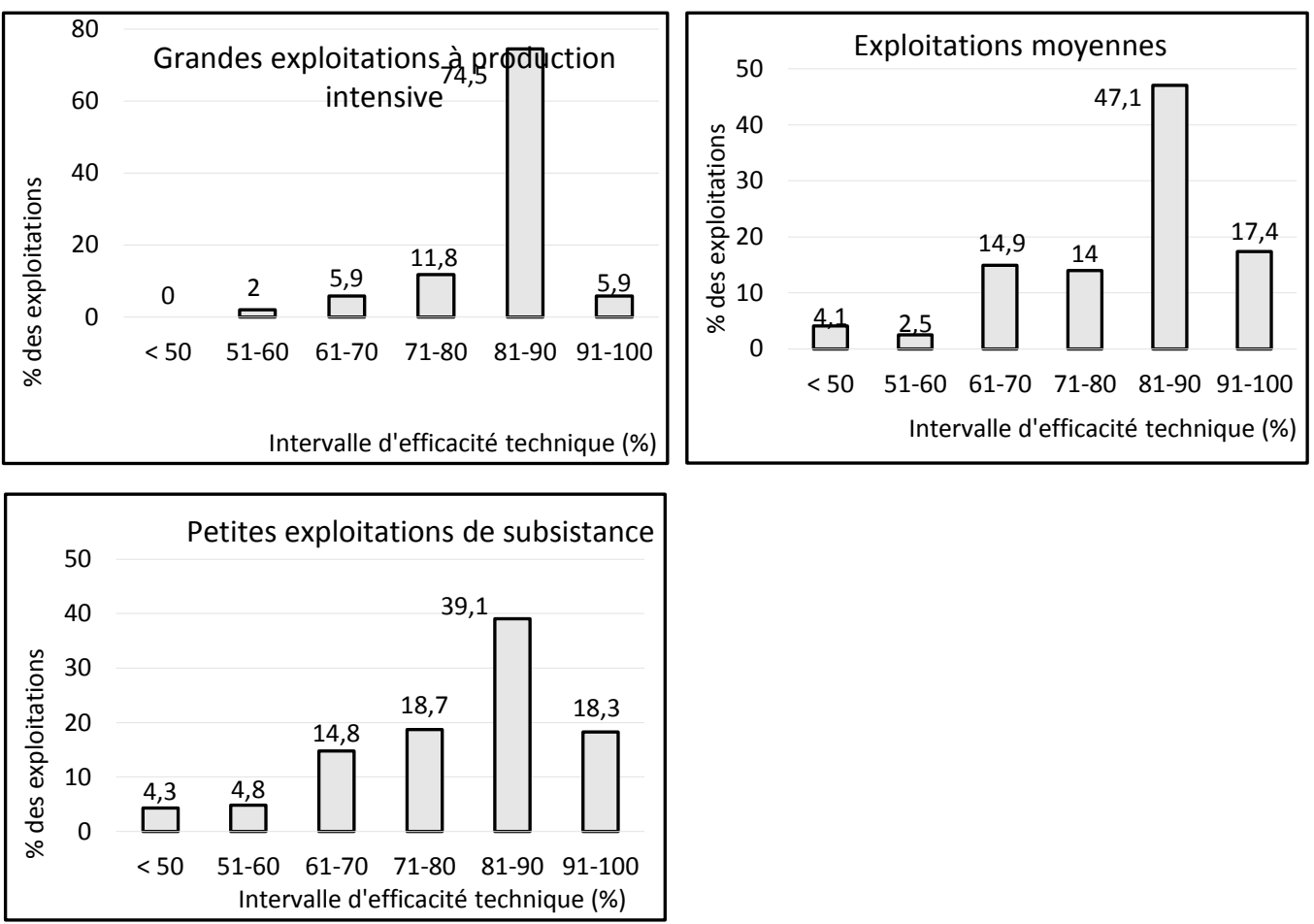

Lorsqu'on observe la structure de la distribution d'efficacité du système des "Grandes exploitations à production intensive", on constate que la plus grande concentration des exploitations (74,5\%) s'observe dans l'intervalle de 81 à $90 \%$ d'efficacité technique, suivi de l'intervalle de 71 à $80 \%$ avec $11,8 \%$.

Dans le système d'"Exploitations moyennes", la majorité des producteurs $(64,5 \%)$ a pu dépasser la barre de $81 \%$ d'efficacité technique, 
avec $47,1 \%$ dans l'intervalle de 81 à 90\% d'efficacité. Néanmoins, il existe encore des augmentations potentielles à travers le besoin de renforcement des capacités de ses producteurs.

$\mathrm{Au}$ niveau du système des "Petites exploitations de subsistance", $57,4 \%$ de producteurs ont pu dépasser la barre de $81 \%$ d'efficacité technique, avec essentiellement 39,1\% dans l'intervalle de 81 à $90 \%$ d'efficacité. Dans ce système, $18,7 \%$ des producteurs seulement ont une efficacité technique comprise entre 71 et $80 \%$, et $23,9 \%$ de producteurs se distribuent de 0 à $70 \%$ d'efficacité technique.

\section{Analyse des déterminants de l'inefficacité technique de la production de maïs}

Bien souvent, l'inefficacité est expliquée par certains facteurs socioéconomiques, techniques, ou institutionnels, plutôt que par l'irrationalité des producteurs.

Onze variables explicatives sont prises en compte dans la présente analyse. Le tableau 4 présente les analyses faites sur ces variables.

Tableau 5. Déterminants de l'efficacité technique de la production du maïs.

\begin{tabular}{|c|c|c|c|c|c|c|}
\hline \multirow[b]{2}{*}{ Variables } & \multirow[b]{2}{*}{ Description } & \multirow{2}{*}{$\begin{array}{l}\text { Signes } \\
\text { attendus }\end{array}$} & \multirow{2}{*}{$\begin{array}{l}\text { Signes } \\
\text { obtenus }\end{array}$} & \multicolumn{3}{|c|}{ Efficacité technique $(T E)$} \\
\hline & & & & Coefficients & $\begin{array}{l}\text { Erreurs- } \\
\text { type }\end{array}$ & Probabilités \\
\hline Sex & Sexe & $+/-$ & + & 0,04 & 0,12 & 0,009 \\
\hline Age & Age & $+/-$ & - & 0,00 & 0,00 & 0,530 \\
\hline Instru & Instruction & + & + & $-0,11$ & 0,08 & 0,057 \\
\hline Alpha & Alphabétisation & $+/-$ & + & 0,11 & 0,08 & 0,273 \\
\hline Mecas & $\begin{array}{l}\text { Appartenance à un } \\
\text { groupement }\end{array}$ & $+/-$ & - & 0,05 & 0,09 & 0,621 \\
\hline Visiteagro & $\begin{array}{l}\text { Encadrement } \\
\text { technique }\end{array}$ & + & + & $-0,00$ & 0,00 & 0,042 \\
\hline Acsem & $\begin{array}{l}\text { Accès aux } \\
\text { semences }\end{array}$ & + & + & $-0,13$ & 0,08 & 0,468 \\
\hline Acintr & $\begin{array}{l}\text { Accès aux } \\
\text { herbicides et } \\
\text { engrais }\end{array}$ & + & + & $-0,19$ & 0,08 & 0,006 \\
\hline Acred & Accès au crédit & + & + & $-0,02$ & 0,09 & 0,088 \\
\hline Tranimal & $\begin{array}{l}\text { Utilisation de la } \\
\text { traction animale }\end{array}$ & + & + & $-0,06$ & 0,15 & 0,000 \\
\hline Tracteur & $\begin{array}{l}\text { Utilisation du } \\
\text { tracteur }\end{array}$ & + & + & $-2,59$ & 1,29 & 0,000 \\
\hline
\end{tabular}

Source : Données de l'enquête (2013).

Les signes positifs attendus et observés pour les variables accès aux semences, accès aux herbicides et engrais, utilisation de la traction animale, et utilisation du tracteur signifient que ces variables sont déterminantes pour l'efficacité de la culture de maïs. Seul l'accès aux semences n'est pas 
significatif, l'accès aux engrais et aux herbicides est significatif à $1 \%$; les autres variables (utilisation de la traction animale et du tracteur) sont hautement significatives à $1 \%$. En effet, cela est prouvé par les critères de caractérisation des systèmes de production où, les exploitants ayant ces matériels comme outils de travail, ont excellé dans la production de maïs en atteignant une meilleure efficacité technique.

\section{Discussion}

Le concept de système de production retenu dans le présent article est celui de Ferraton et Touzard (2009) qui s'inspirent eux-mêmes de Reboul (1976). En effet, pour Reboul (op. cit.), « un système de production correspond à une association spécifique de systèmes de culture et d'élevage, mise en œuvre par les agriculteurs en fonction des parcelles disponibles et de leur localisation, des équipements utilisés, de la force de travail familiale ou mobilisable, et appliquée à un ensemble d'exploitations ».

Pour évaluer les performances techniques des systèmes de production en vue d'éclairer leur fonctionnement, Ferraton et Touzard (2009) ont comparé la valeur ajoutée brute par actif et par journée de travail dans différents systèmes de culture. Cette approche permet, selon les auteurs, avant tout de comprendre comment se font les choix d'affectation des ressources disponibles.

$\mathrm{Au} \mathrm{vu} \mathrm{des} \mathrm{résultats} \mathrm{obtenus,} \mathrm{la} \mathrm{présente} \mathrm{recherche} \mathrm{estime} \mathrm{que} \mathrm{le}$ niveau technologique est relativement satisfaisant pour l'ensemble des trois systèmes de production à base de maïs. Ils montrent également que pour les maïsiculteurs de la zone de recherche, il existe encore des gains potentiels considérables à réaliser sur les quantités de facteurs de production en maintenant constants les niveaux actuels de production ou autrement, qu'il existe encore d'énormes marges de manœuvres pour accroître la production maïzicole sur la base des ressources actuellement utilisées. Cependant, qu'il existe des possibilités d'accroissement de l'efficacité technique de 10 à $20 \%$ sans utiliser d'intrants supplémentaires.

Contrairement à ce résultat, Houndétondji et al. (2014), ont trouvé que les producteurs de maïs au Bénin sont techniquement inefficaces. Selon ces auteurs, les producteurs n'ayant pas intégré l'utilisation des engrais chimiques dans leur système de production sont plus efficaces que ceux qui les utilisent.

Selon Balcombe et al, (2008) ainsi que Battese et Coelli (1995), les déterminants de l'efficacité technique généralement utilisés en agriculture sont le crédit, l'encadrement agricole, le nombre d'années d'expérience de l'exploitant, son âge, son niveau d'instruction, la taille de l'exploitation et l'entraide, etc. 
Selon Houndétondji et al. (2014), quatre variables influencent significativement l'efficacité technique des producteurs de maïs. Les auteurs ont noté que les variables sexe, densité agricole, et pratique d'activités extraagricoles affectent positivement l'efficacité technique, et que la variable superficie emblavée agit négativement sur elle. Ils en déduisent que plus les unités de production de maïs sont dirigées par les hommes, plus elles sont efficaces techniquement, du fait de la facilité de leur contact avec les agents d'encadrement et de leur facilité d'accès à la main-d'œuvre familiale ou de moyens financiers pour engager la main-d'œuvre salariée afin de réaliser les différentes opérations culturales. Ce que confirment nos résultats.

L'analyse des déterminants de l'efficacité technique révèle que les variables sexe, niveau d'instruction et alphabétisation de l'exploitant sont de signe positif, mais seul le déterminant sexe est significatif à $5 \%$. En revanche, les variables âge, instruction, alphabétisation et appartenance à une association coopérative de producteurs de maïs n'ont aucune influence significative sur l'efficacité technique des exploitations de maïs enquêtées. De plus, l'âge et l'appartenance à un groupement coopératif sont de signe négatif. L'analyse de ces résultats révèle donc que les hommes produisent plus et sont plus efficaces que les femmes dans la production de maïs. L'explication qui pourrait y ressortir est que vu les étendues des superficies et les types d'équipements mis en œuvre pour la production, les femmes ne sont pas souvent favorisées par les événements (accès à la terre, investissements, opérations de labour etc.). Le signe positif porté par les niveaux d'instruction ou d'alphabétisation des producteurs confirme l'influence positive que pourraient avoir ces variables sur l'efficacité de l'exploitant. L'appartenance à un groupement n'est pas ici une nécessité en production de maïs car cette denrée est vendue sur le marché en indépendant.

La variable relative à l'alphabétisation n'est pas du tout significative, cela montre que l'alphabétisation n'est pas mise au profit de la production de maïs, probablement parce que les échanges sont réalisés en langue locale tandis que les documents de vulgarisation ou des fiches techniques sur la production de maïs pouvant permettre aux producteurs d'acquérir le savoir et le savoir-faire sont tous écrits en français. Au même moment, malgré son signe positif, la variable instruction n'est pas significative. En effet, les producteurs instruits sont pour la plupart des fermiers résidant en ville. Ils embauchent des ouvriers non lettrés à qui ils confient les travaux de production. Par conséquent, leur instruction et leur savoir ne sont pas mis au service de la production du maïs.

Suite aux déclarations des producteurs, l'accès aux semences ici ne constitue pas une préoccupation. Bien que la semence constitue le deuxième facteur de production après la terre, seuls les producteurs du $2^{\text {ème }}$ type de système "Exploitations moyennes" utilisent les semences améliorées. Par 
ailleurs, les herbicides qui viennent suppléer la main-d'œuvre lors des différents sarclages, accélèrent les travaux surtout pour les producteurs ayant des grandes superficies, ou pour ceux qui ont d'autres cultures secondaires pour lesquelles ils doivent libérer la main-d'œuvre du champ de maïs à temps. Cependant, nombreux sont les producteurs qui n'ont pas accès à l'herbicide, cet intrant n'étant pas disponible au niveau des canaux officiels ; et pour ceux qui en disposent, ils l'ont acquis au marché noir. Le prix de revient est alors prohibitif, et son utilisation est réservée pour les élites (producteurs des deux classes extrêmes), donc les plus nantis.

Les variables fréquemment testées dans les études de déterminants d'efficacité technique sont l'encadrement technique et l'accès au crédit. Ces deux variables sont significatives, avec l'encadrement technique au seuil de $5 \%$, et l'accès au crédit à $1 \%$. L'encadrement technique est une variable importante pour l'efficacité et le signe positif attendu et obtenu implique que les producteurs qui reçoivent un nombre élevé de visites d'agent d'encadrement sont techniquement plus renforcés. Les rencontres avec les agents de vulgarisation sont un moyen pour les producteurs de discuter des difficultés qu'ils rencontrent dans leurs pratiques culturales quitte à ce que des solutions sous-forme de conseils ou de nouvelles technologies leur soient apportées. Ainsi, plus le producteur expose ses contraintes, plus il acquiert des connaissances et plus il est techniquement efficace s'il les applique.

Il est à noter que dans notre échantillon, 72,7\% des producteurs affirment n'avoir l'assistance ou la visite des agents de vulgarisation qu'une seule fois l'an; paradoxalement, ces producteurs sont ceux qui ont le plus besoin d'encadrement car ils sont essentiellement de la classe des petits producteurs moins nantis, et dont les productions sont très faibles. Il résulte donc que pour augmenter l'efficacité de ces producteurs, le dispositif d'encadrement soit renforcé afin d'accroître les fréquences des visites aux producteurs.

Les effets du crédit et de l'instruction sur la productivité des agriculteurs ont fait l'objet de controverses; pour certains chercheurs, ils améliorent la performance agricole. Pour d'autres, ils n'ont aucun effet sur l'efficacité technique des producteurs agricoles.

Les analyses ont montré que l'obtention du crédit ne se révèle pas être significative au sein des trois systèmes de production étudiés mais reste positive dans la production du maïs. Ceci s'explique par le fait que non seulement le crédit n'est pas disponible, mais surtout que les conditions d'octroi du crédit agricole ne sont pas encore réunies au Bénin. Par ailleurs, l'instruction a été évaluée en termes de niveau d'études ; là c'est une variable qui devrait améliorer l'efficacité des exploitants agricoles dans la mesure où un producteur agricole instruit, a facilement la maîtrise des techniques modernes de production (Battese et Coelli, 1995; Coelli et Fleming, 2004), 
mais dans la présente, nous avons trouvé le contraire. En effet, les plus instruits dans notre échantillon ont d'autres activités plus rémunératrices, et confient les travaux agricoles à des personnes moins instruites, qui n'ont d'autre salut que l'agriculture. Ces personnes sont alors soit des ouvriers agricoles parfois mal rémunérés, ou même des personnes abandonnées dans des travaux serviles. Par conséquent, la production de maïs en souffre. Cependant, Midingoyi (2008) a argumenté que les producteurs qui sont instruits ont un niveau d'efficacité meilleur. Cela s'explique pour le cas du coton car il constitue la seule culture locomotive au Bénin, culture organisée en filière ; et la plupart des producteurs de maïs n'hésitent pas à se réfugier derrière la culture de coton pour obtenir l'appui technique et des intrants auprès de l'administration d'encadrement agricole. Il n'est donc pas rare dans les zones agroécologiques du Nord et du Centre-Bénin, de constater que la culture du maïs entre dans la rotation du coton; son devenir est ainsi lié à celui du coton dont il profite de la fertilisation.

Pour durer, les systèmes de production doivent trouver en eux-mêmes un équilibre agroécologique, économique et social. Cet équilibre peut aussi être partiellement trouvé au moyen d'une fusion de systèmes complémentaires interagissant entre eux par des échanges de matières, de moyens de production et d'informations. Il convient alors d'envisager l'adaptation des outils de mécanisation tels que la traction animale dans les autres localités où persistent les systèmes moins performants.

\section{Conclusion et implications}

Dans la présente recherche, en vue de vérifier l'hypothèse émise, l'évaluation des niveaux d'efficacités techniques des systèmes de production de maïs a été faite. Elle a permis de mettre en relief les niveaux d'efficacités techniques actuels des systèmes de production de maïs au Bénin, et les réserves dont ils disposent encore pour accroître leur productivité. Pour y parvenir, des régressions ont été effectuées grâce aux programmes SPSS, STATA, FRONTIER 4.1c, et aux logiciels Access, Excel et Word. Les résultats ont montré que le niveau technologique est relativement satisfaisant pour l'ensemble des trois systèmes de production à base de maïs mis en compétition, mais qu'il existe des possibilités d'accroissement de l'efficacité technique au sein de ces systèmes de production au Bénin. Ces résultats suggèrent que, toutes choses étant égales par ailleurs, les exploitations n'utilisant pas le machinisme agricole sont les moins efficaces. Ceci justifie le résultat obtenu concernant l'excès d'utilisation de la main-d'œuvre salariée.

Les contraintes sont en général les mêmes d'une localité à une autre. Les principales contraintes sont la baisse de la fertilité des sols, l'insuffisance des ressources financières, la non-disponibilité des intrants, etc. Les 
différentes politiques régionales et nationales mises en œuvre, devront alors contribuer à réduire les écarts entre le niveau maximum de production que l'on peut obtenir et son niveau réel. Pour améliorer l'efficacité technique de la production de maïs au Bénin, les actions idoines doivent être engagées vers le renforcement du dispositif d'encadrement technique pour la maitrise et le respect des itinéraires techniques de cette culture surtout en direction des femmes et des exploitants pauvres, la promotion de la mécanisation progressive à travers le crédit agricole afin de surmonter les difficultés d'accès à la main-d'œuvre.

D'un point de vue politique, il est intéressant de rechercher les sources de l'inefficacité et d'identifier les déterminants des performances techniques. Les pouvoirs publics peuvent agir sur les déterminants ainsi identifiés pour améliorer l'efficacité globale tout en réduisant l'écart de productivité entre les zones.

\section{References :}

Adégbola Y.P., Oloukoï L., Houéssionon P., Kinkpé A.T., Adjovi D., Bankolé A-B., Foundohou A. (2013). Comptes économiques de l'agriculture (CEA) du Bénin. Volume 1 : Rapport synthèse, avril 2013, 118p.

Ahouannenou G.L., Houenou B. (2011): Commerce et concurrence transfrontaliers du maïs : Etude sur opportunités et transparence des marchés et analyse des risques et coûts de transaction. Fédération des Unions des Producteurs du Bénin (FUPRO-BENIN), 54p.

Amemiya T. (1984). Modèles de Tobit : un aperçu. Journal de l'économétrie, 24(1-2), 3-61.

ARAA (2009). Les systèmes de culture durables : 1'expérimentation de long terme au service de l'agriculture durable. Compte rendu des essais systèmes, $2 \mathrm{p}$.

Balcombe K., Fraser I., Latruffe L, Rahman M., Smith I. (2008). Examining sources of technical efficiency in Bangladesh rice farming: An application of a double bootstrap. Applied Economics, vol. 40 (15), p. 1919-1925.

Battese G.E., Coelli T.J. (1995). A model of technical inefficiency effects in a stochastic frontier production function for panel data. Empirical Economics, vol. 20, p. 325-332.

BCEAO (2012). Rapport sur la situation de la pauvreté dans les pays de l'UEMOA : « PNUD et Développement humain », 38p.

Biaou G. et Gnimadi C. (2012). Étude socio-économique de synthèse des villages de Founougo_A, Founougo_B et Kanderou. Rapport provisoire pour le "Projet d'Appui à l'Opérationnalisation des Villages du Millénaire" (PVM). Avril 2012, 150p. 
Blancard S., Boussemart J-P., Flahaut J., Lefer H-B. (2013). Les fonctions distances pour évaluer la performance productive d'exploitations agricoles. Économie rurale, $\mathrm{n}^{\circ} 334$.

Butault J-P. (2006). La formation des revenus agricoles dans les différentes orientations entre 1990 et 2004. Communication à la Commission des Comptes de l'Agriculture de la Nation. Paris, 26 juin 2006.

Coelli T., Fleming E. (2004). Diversification economies and specialization efficiencies in a mixed food and coffee smallholder farming system in Papua New Guinea. Agricultural Economics, vol. 31, p. 229-239.

Coelli T.J. (1996). A guide to FRONTIER Version 4.1: A Computer Program for Stochastic Frontier Production and Cost Function Estimation. CEPA working papers Department of Econometrics University of New England Armidale, NSW 2351, Australia. 33p.

Coelli T.J., Prasada Rao D.S., Battese G.E. (1998). An introduction to efficiency and productivity analysis. Kluwer Academic Publishers, Boston/Dordrecht/London, 274p.

Coelli T.J., Prasada Rao D.S., O’Donnell C.J. and Battese G.E. (2005). An Introduction to Efficiency and Productivity Analysis. Kluwer Academic Publishers, Boston. Dordrecht, London, $2^{\text {nd }}$ edition, 2005, XVII, $341 p$.

Ferraton N. et Touzard I. (2009). Comprendre l'agriculture familiale : diagnostic des systèmes de production. Éditions Quce, CTA, Presses agronomiques de Gembloux-2, Passage des Déportés 5030 Gembloux, Belgique, 135p.

Ghali M., Daniel K., Colson F. et Latruffe L. (2014). Diagnostic de l'efficacité technique des exploitations agricoles françaises : une analyse de l'efficacité d'utilisation des ressources énergétiques et exploration des déterminants relevant des pratiques agricoles, $29 \mathrm{p}$.

Houndétondji D.S., Biaou G., et Zannou A. (2014). Efficacité technique de la production du maïs dans la commune de Zogbodomey au Bénin. Bulletin de la Recherche Agronomique du Bénin (BRAB). Numéro spécial Economie et Sociologie Rurales-décembre 2014. 1025-2355 et ISSN en ligne (on line) : 1840-7099. Pp 43-51.

INSAE, Bénin (2011). www.africaneconomicoutlook.org/fr, Consulté le 05 juin 2015.

Latruffe L. (2010). Compétitivité, productivité et efficacité dans les secteurs agricole et agroalimentaire. Éditions OCDE. http://dx.doi.org/10.1787/5km91nj6929p-fr. Accédé le 21 juillet 2015.

Midingoyi G. S-K (2008). Analyse des déterminants de l'efficacité de la production cotonnière au Bénin : Cas des départements de l'Alibori et de l'Atacora. Mémoire de Master complémentaire en économie et sociologie rurales. Académie Universitaire de Wallonie-Europe. Gembloux, 90p. 
Neyman J. (1934). On the two different aspects of representative method: the method of stratified sampling and the method of purposive selection. Journal of the Royal Statistical Society, Vol. 97, n 4 (1934), pp. 558-625.

PNUD, BAfD, OCDE, CEA (2011). Perspectives économiques en Afrique, Bénin 2011. 18p.

Ray S.C. (2004). Data Envelopment Analysis: Theory and techniques for economics and operation research. Cambridge University Press, 353 pages.

Sadoulet E and De Janvry A. (1995). Quantitative Development Policy Analysis: Exercise Solutions, Volume 4, Johns Hopkins University Press, $65 \mathrm{p}$.

Schnedler W. (2005). Évaluation de probabilité pour des vecteurs aléatoires censurés. Revues économétriques, 24(2), 195-217.

Tobin J. (1958). Estimation of relationships for limited dependent variables, Econometrica (The Econometric Society) 26 (1): 24-36. 